\title{
A micro optical probe for edge contour evaluation of diamond cutting tools
}

\author{
S. H. Jang, Y. Shimizu, S. Ito, and W. Gao \\ Department of Nanomechanics, Tohoku University, Sendai, Japan \\ Correspondence to: Y. Shimizu (yuki.shimizu@nano.mech.tohoku.ac.jp)
}

Received: 31 October 2013 - Accepted: 4 March 2014 - Published: 28 March 2014

\begin{abstract}
This paper presents a micro optical probe, which is employed to evaluate edge contours of single point diamond tools with a size in a range of several millimetres. The micro optical probe consists of a laser source with a wavelength of $405 \mathrm{~nm}$, an objective lens with a numerical aperture of 0.25 , a photodiode for measurement, and a compensating optical system including another photodiode for compensation of laser intensity. A collimated laser beam, which is divided by a beam splitter in the compensating optical system, is focused by the objective lens so that the focused spot can be used as the micro optical probe. The micro optical probe traces over an edge contour of an objective tool while the signals of both the two photodiodes are monitored. The output of the photodiode for measurement is compensated by using that of the photodiode for laser intensity compensation to eliminate the influence of the laser instability. The signal of the photodiode for measurement is used to define the deviation of edge contour within the diameter of the micro optical probe. To verify the feasibility of the developed optical probe, the optical system was mounted on a diamond turning machine, and some experiments were carried out. Two types of edge contours of the diamond tools having a straight cutting edge and a round cutting edge were measured on the machine.
\end{abstract}

\section{Introduction}

Ultra-precision machining technology has enabled us to generate mirror surfaces by direct cutting (Moriwaki and Okuda, 1989; Evans and Bryan, 1999; Brinksmeier et al., 2010). The applications of ultra-precision machining have also been expanded by enhancements of the machining accuracy with error compensation methods (e.g. Kong et al., 2008). For ultraprecision machining, single point diamond cutting tools with a nose radius on the order of several $10 \mathrm{~nm}$ are often employed (Zong et al., 2010). In the fabrication of precision components such as optics and functional surfaces with micro patterns, the form error of the tool's cutting edge is one of the critical factors that will influence the quality of the machined surfaces (Brehm et al., 1979; Li et al., 2005; Lane et al., 2010). The form of the tool's cutting edge should therefore be evaluated periodically to assure the machining accuracy (Jiang, 2011; Goel, et al., 2013). In addition, onmachine measurement of the tool's cutting edge is strongly desired because a removal of the cutting tool from the ma- chine would induce a certain amount of tool misalignment when carrying out the tool installation again. Even if such an installation error is small, it should be taken seriously in cases of ultra-precision machining (e.g. Chen et al., 2010; Fang et al., 2013).

One of the conventional methods to measure the form of a cutting tool is by scanning electron microscopes (SEMs) (Drescher, 1993; Asai et al., 1990). However, quantitative analysis of the tool's form by using the acquired SEM images, which are qualitative results, is expensive and timeconsuming task (Lane, et al., 2013). In addition, the SEMs cannot be applied to on-machine tool measurement because the tool should be unmounted from the machine so that the evaluation can be carried out in a vacuum condition.

Atomic force microscopes (AFMs) are appropiate instruments to measure the tool's cutting edge (Lucca and Seo, 1993; Krulewich Born and Goodman, 2001; Zong et al., 2007). The AFMs have several advantages such as three-dimensional (3-D) imaging, sub-nanometric measurement resolution and low measuring force. An AFM-based 
measuring instrument, which is appropriate for on-machine measurement, has been proposed to evaluate the form of the tool's cutting edge (Gao et al., 2006). In the proposed instrument, an optical alignment system has been employed to align the AFM probe to the tool's cutting edge (Jang et al., 2011) instead of using optical microscopes on the commercial AFMs. The proposed instrument has realized onmachine quantitative measurement of the tool's cutting edge (Gao et al., 2009). However, due to the limited measurement range of the AFM system, it takes a long measuring time for form measurements of a large-scale cutting tool.

In this paper, the optical alignment system developed in the AFM-based measuring instrument is proposed to be used as a micro optical probe for rapid and quantitative measurement of large-scale cutting edge of tools with a size in a range of several millimetres. In order to evaluate the tool's edge contours, a focused beam spot is employed as the micro optical probe. In the proposed measurement method, deviation of the intensity of the light, which is a part of the optical probe passed through the tool's edge, would be converted into the information of the tool's edge contour. The principle of the proposed method and an attempt to improve the measurement resolution of the micro optical probe are described. In addition, the edge contours of both a straight-shaped cutting tool and a round-shaped cutting tool are measured by the developed on-machine measurement system.

\section{Tool contour measurement with a micro optical probe}

\subsection{Proposed measurement method}

A micro optical probe has been developed to measure edge contours of single point diamond tools. A schematic of the proposed micro optical probe is shown in Fig. 1. A rake face of the diamond tool with a large-scale edge contour is set perpendicular to the optical axis of the probe. In this paper, a focused laser beam by the objective lens is called a micro optical probe and is used for measurement of edge contours. The intensity of the laser beam, which is a part of the optical probe passed through the tool edge, is collected by a photodiode (PD). The voltage signal of a current-to-voltage circuit, which converts photoelectric current output of the PD to a voltage signal, is referred to as the PD output. A relationship between the PD output and the $y$-directional position of the optical probe with respect to the tool's edge is shown in Fig. 1b. By referring the relationship curve in Fig. 1b, the PD output can be converted to the relative position of the probe with respect to the tool's edge contour. When the information on the absolute probe position is available from the tool positioning system, the edge contour can therefore be acquired from the deviation of the PD output and the information on the absolute probe position; that is the core principle of the proposed method.

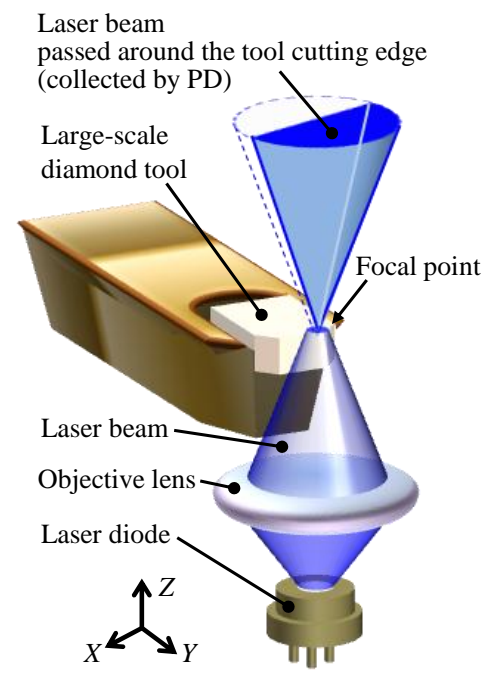

(a)

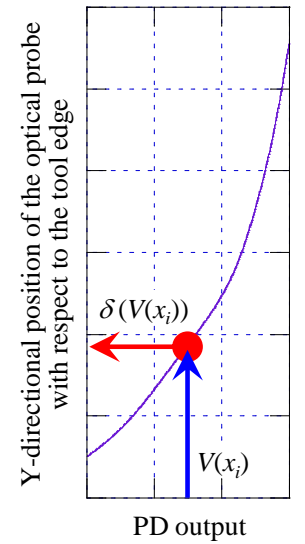

(b)
Figure 1. Schematic of the proposed micro optical probe. (a) Configuration of the optical setup. (b) Relationship between the PD output and the $y$-directional position of the optical probe with respect to the tool's edge.

Figure 2 shows a schematic of how the PD output can be converted to the relative position of the probe with respect to the tool's edge. In Fig. 2, $h\left(x_{i}\right)\left(=\delta\left(V\left(x_{i}\right)\right)\right)$ is the relative position of the centre point of the optical probe with respect to the tool's edge, $g(x)$ is a scanning path of the micro optical probe. The tool's edge contour $f(x)$ can be expressed as

$f(x)=g(x)+h(x)$.

While the optical probe is tracing the scanning path $g(x)$, both the PD output $V\left(x_{i}\right)$ and the probe position $g\left(x_{i}\right)$ at each $x$ position $\left(x_{i}, i\right.$ : sampling number) are recorded simultaneously. After that, by referring the relation curve shown in Fig. 1b, the PD output $V\left(x_{i}\right)$ can be converted to the relative position $h\left(x_{i}\right)\left(=\delta\left(V\left(x_{i}\right)\right)\right)$.

Figure 3 shows a schematic of the alignment of the tool's edge with respect to the optical probe. In the proposed method, the tool's edge should be aligned to the optical stylus precisely in the $X Y$ plane. In addition, the tool should also be positioned at the beam's waist as shown in Fig. 3a. The variation of the PD output with respect to the tool displacement along the $z$ direction is shown in Fig. 3b. The PD output varies as the change of the sectional size of the beam, and has minimum value when the tool is positioned at the beam's waist of the optical probe. In the proposed method, the $z$-directional alignment can thus be carried out by monitoring the PD output before the probe scanning in the $X Y$ plane. The measurement resolution of the proposed method is mainly determined by the accuracy of the scanning motions and spot size of the optical probe, and $S / N$ (signal-tonoise) ratio of the PD output. It should be noted that the beam 


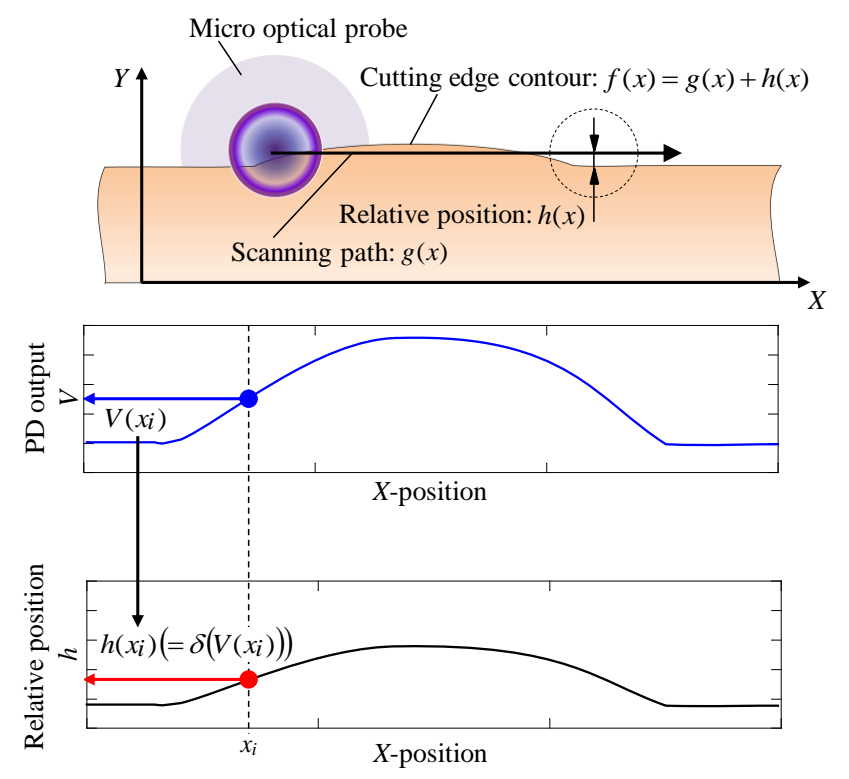

Figure 2. A schematic of how the PD output can be converted to the relative position of the probe with respect to the tool's edge.

spot is small enough to be treated as a "point" on the cutting edge in terms of the tool's nose radius.

\subsection{Measurement system}

In order to measure form errors and radii of tool edge contours, a measurement system including an optical system for the micro optical probe has been developed. Figure 4 shows a schematic of the developed system. The optical probe consists of a laser diode (LD), a beam splitter (BS), three lenses and two PDs. The optical system is mounted on a diamond turning machine, which has a precision positioning system along the $\mathrm{z}, \mathrm{y}$, and $\mathrm{z}$ directions. A laser beam, which has a wavelength of $405 \mathrm{~nm}$, is emitted from the LD along the $z$ direction, and is collimated by a collimator lens. The collimated laser beam is divided into a reference beam and a measurement beam by using the BS. Deviation of the light intensity of the reference beam is monitored by the PD for reference $\left(\mathrm{PD}_{\mathrm{R}}\right)$ so that the influence of the LD power drift and some noises due to electric field around the LD can be compensated. Meanwhile, the measurement beam is focused on the tool's edge by an objective lens. Both the collimator lens and the objective lens are aspherized achromatic lenses with a numeric aperture (NA) of 0.25 . The laser beam spot has a diameter of $2 \mu \mathrm{m}$ at the beam waist (defined by a full width at half maximum). The laser beam's spot is employed as the micro optical probe in this paper. The size of the focused beam is mainly governed by the NA of the objective lens and the laser wavelength $\lambda$. The spot radius $r_{0}$ at the diffraction limit edge is expressed as follows (Grosjean and Courjon, 2006):

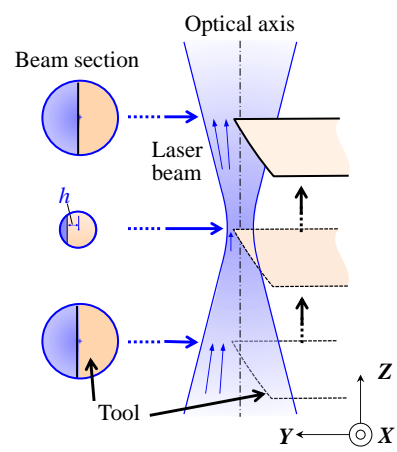

(a)

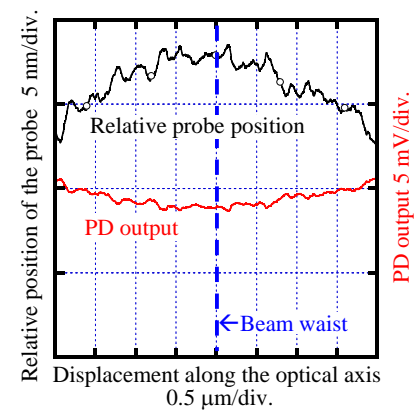

(b)
Figure 3. A schematic of the alignment of the tool's edge with respect to the optical probe. (a) Schematic of the tool positioning. (b) Relation curves for PD output, displacement along the $z$ direction and the relative probe position converted from PD output.

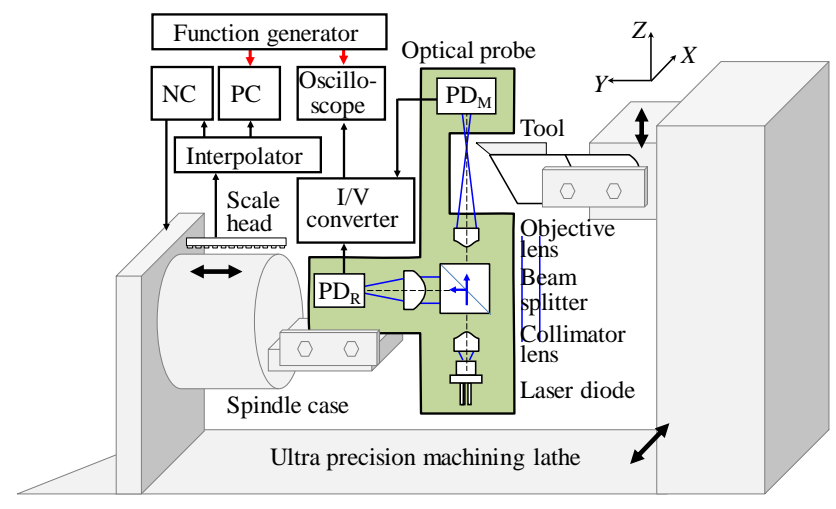

Figure 4. A schematic of the on-machine tool's edge contour measurement system.

$r_{0} \propto \frac{\lambda}{\mathrm{NA}}$

According to Eq. (2), an objective lens with a higher NA generates a smaller laser beam spot that can achieve a higher resolution. However, attention should be paid for the lightcollecting part of the micro optical probe when applying the objective lens with a high NA because the light passed through the tool's edge would propagate rapidly.

A portion of the direct beam that passed through the tool's edge is captured by the $\mathrm{PD}$ for measurement $\left(\mathrm{PD}_{\mathrm{M}}\right)$ during the scanning of the micro optical probe. The output of $\mathrm{PD}_{\mathrm{M}}$ is used as a measurement signal. In this paper, the $\mathrm{I}-\mathrm{V}$ circuit for both $\mathrm{PD}_{\mathrm{M}}$ and $\mathrm{PD}_{\mathrm{R}}$ are arranged on the same electric circuit board so that influences of circuit power instability and noises due to electric field, which would affect signal quality of the PDs, can be minimized.

Figure 5 shows a photograph of the developed optical setup for the micro optical probe mounted on the diamond turning machine. To carry out on-machine measurement, the 


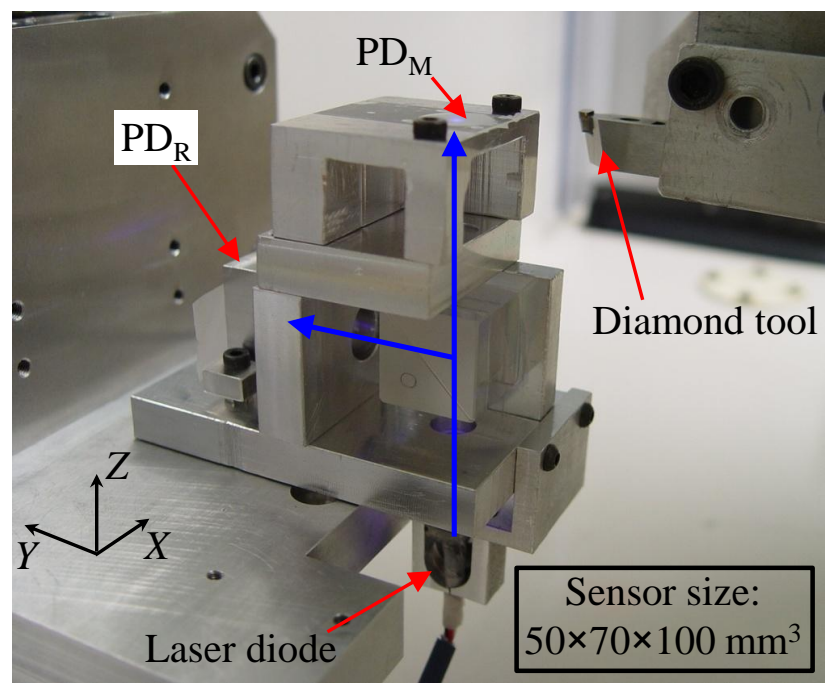

Figure 5. The developed micro optical probe mounted on the diamond cutting machine.

optical setup was mounted on the casing of the spindle of the diamond turning machine, while a diamond tool was mounted on the $x y z$ carriage slides of the turning machine. The carriage slides have a nanometre-order positioning resolution with repeatability of less than $\pm 50 \mathrm{~nm}$ in both the $x$ and $y$ directions, and can be used for the accurate positioning and scanning of the optical probe. Both the $x y z$-directional position of the optical probe and the PD outputs were captured simultaneously during the tracing of the optical probe over the tool's edge.

\section{Experiments}

\subsection{Stability of the developed optical probe}

Stability of the developed micro optical probe was investigated in experiments. The developed optical system was mounted on the diamond turning machine as shown in Fig. 5, and the PD outputs were captured while the optical probe was kept stationary on the tool's edge. At first, the BS was removed from the optical setup, and the variation of $\mathrm{PD}_{\mathrm{M}}$ output $\left(V_{\mathrm{M}}\right)$ was monitored. Figure 6a shows the measured output waveform of the $\mathrm{PD}_{\mathrm{M}}$. The amplitude of the $\mathrm{PD}_{\mathrm{M}}$ output was found to be about $40 \mathrm{mV}$ while the one with a simple moving average (window size: $10 \mathrm{~ms}$ ) was about $10 \mathrm{mV}$. After that, the BS was integrated into the optical setup again, and variations of both the $\mathrm{PD}_{\mathrm{M}}$ output and $\mathrm{PD}_{\mathrm{R}}$ output $\left(V_{\mathrm{R}}\right)$ were monitored. Figure $6 \mathrm{~b}$ shows the measured PD outputs. The amplitudes of both the $\mathrm{PD}_{\mathrm{M}}$ and $\mathrm{PD}_{\mathrm{R}}$ outputs were about $50 \mathrm{mV}$, which was almost the same as the one shown in Fig. 6a. In Fig. 6b, unpredictable variations of the PD outputs, which were mainly due to a drift of LD power, were also observed. In the developed optical system, the influence of the LD instability was minimized by using the following

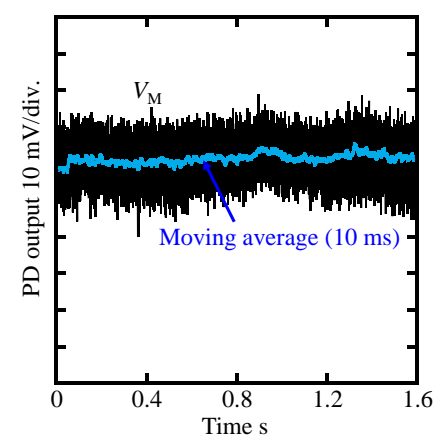

(a)

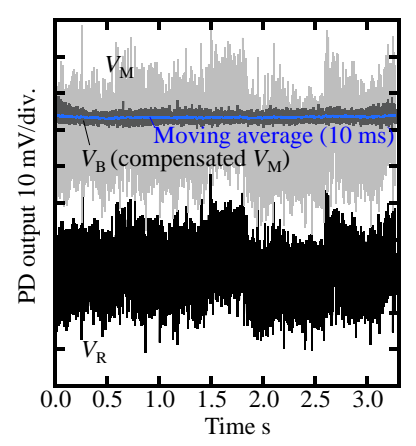

(b)
Figure 6. Stability of the PD outputs: (a) stability without compensation, (b) stability with compensation.

equation:

$V_{\mathrm{B}}=V_{\mathrm{M}} \times \frac{V_{\mathrm{R}_{1}}}{V_{\mathrm{R}}}$

In Eq. (3), $V_{\mathrm{B}}$ is the compensated $\mathrm{PD}_{\mathrm{M}}$ output, and $V_{\mathrm{R}_{1}}$ is an initial $\mathrm{PD}_{\mathrm{R}}$ output. The compensated $\mathrm{PD}_{\mathrm{M}}$ output waveform had the amplitude of about $6 \mathrm{mV}$, in which a noise component with a frequency of $100 \mathrm{~Hz}$ due to the machine tool vibration was found. The amplitude of the $V_{\mathrm{B}}$ with a simple moving average (window size: $10 \mathrm{~ms}$ ) was less than $1.5 \mathrm{mV}$, corresponding to the tool's edge contour of less than $10 \mathrm{~nm}$. From these results, the feasibility of the developed optical system on the improvement of the optical probe stability was verified.

\subsection{Contour evaluation of a straight cutting edge}

A straight cutting edge of a diamond tool was measured to investigate the feasibility of the developed measurement system. The straight cutting edge was aligned to be parallel with the $x$ axis of the measurement system within a tolerance of $1 \mu \mathrm{m}-2 \mathrm{~mm}$ since the positioning error of the micro optical probe along its optical axis would influence the accuracy of the edge contour measurement as shown in Fig. 3b. A scanning speed of the optical probe was set to be $0.267 \mathrm{~mm} \mathrm{~s}^{-1}$. It took about $13 \mathrm{~s}$ to scan the tool's edge with a length of $3.5 \mathrm{~mm}$. The sampling frequency of the PD output was set to be $4 \mathrm{kHz}$. All the measurements were carried out on the machine, under constant temperature conditions of $24.2 \pm 0.1^{\circ} \mathrm{C}$.

Prior to the edge contour measurement, the relationship between the PD output and the relative position was investigated in experiments. The optical micro probe was moved along the $y$ direction while the PD output was monitored. Figure 7 shows the acquired relationship between the PD output $V$ and the relative position $\delta$. The encoder readout of the $y$ axis slide of the diamond turning machine was used to determine the relative position $\delta$. The acquired relation curve in Fig. 7 was fitted by a polynomial function. 


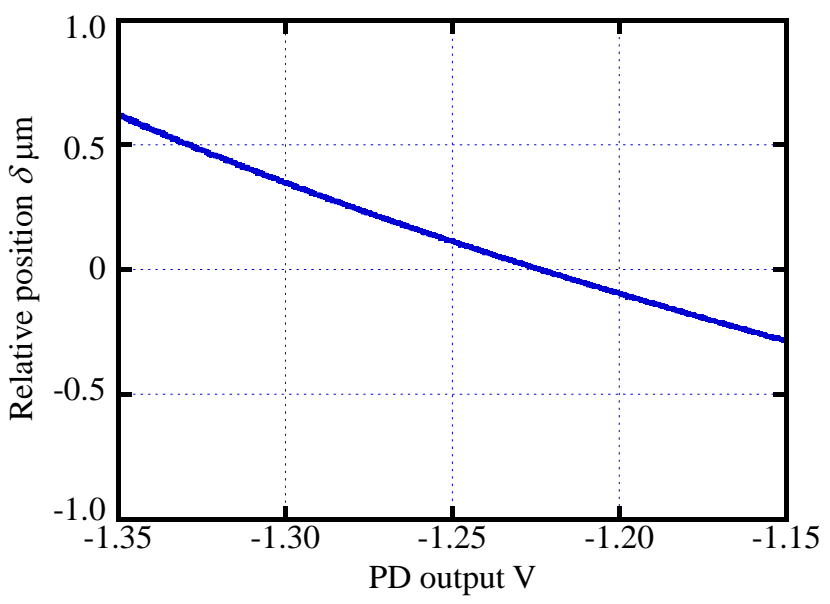

Figure 7. Relationship between the PD output and the $y$-directional relative position of the optical probe with respect to the flat-shaped cutting edge.

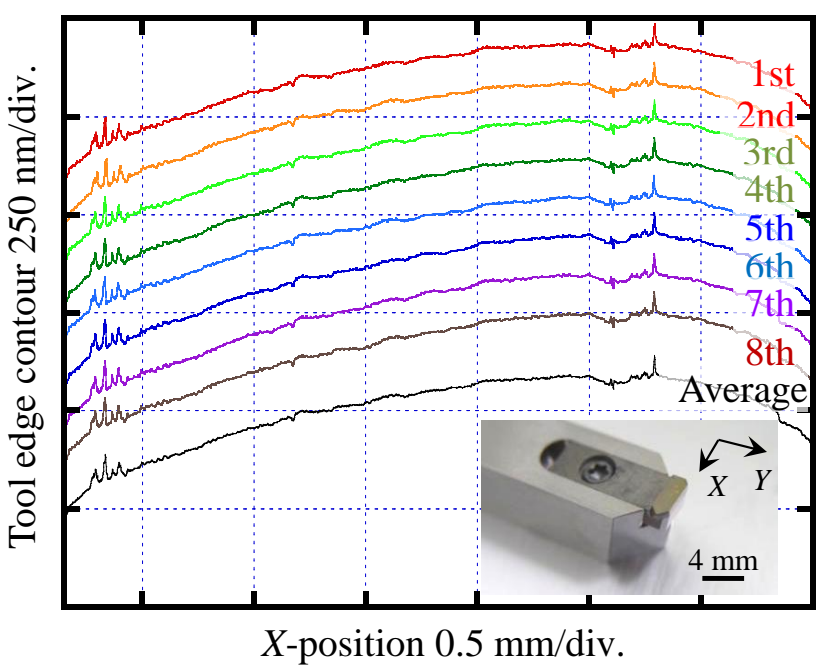

Figure 8. Measured cutting edge contour of the straight-shaped diamond tool.

Figure 8 shows the edge contour measured by the developed system. The PD output was converted into the edge contour by using the relation curve shown in Fig. 7. In Fig. 8, the averaged edge contour acquired by averaging the results of eight time measurements is also plotted. The measurement results were found to agree with each other. A large deviation of $400 \mathrm{~nm}$ from the straight shape over the whole measurement range was observed. Measurement repeatability was investigated by plotting the deviations of each measured edge contour from the averaged one as shown in Fig. 9. At the areas of smooth edge contour, measurement repeatability was found to be below $10 \mathrm{~nm}$. The influence of the mechanical vibration of the system, a frequency which was about $1 \mathrm{~Hz}$, was observed. The measurement repeatability became worse at the area of steeply deviated edge contour. The main reason

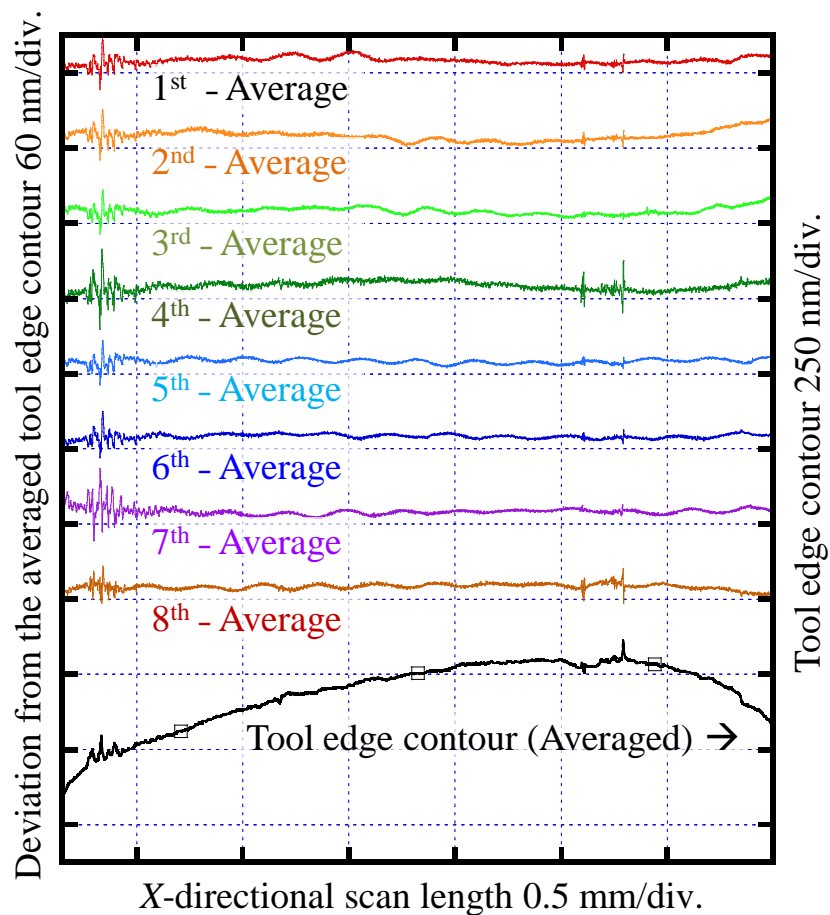

Figure 9. Averaged measurement result of a flat-shaped cutting edge from eight measurements and their deviations.

of this is due to the slight difference of the $x$-directional positioning of the micro optical probe at each measurement. As shown in Figs. 8 and 9, the straight cutting edge having submicron form accuracy was successfully evaluated over the measurement range of $3.5 \mathrm{~mm}$ by the developed measurement system.

\subsection{Contour evaluation of a round cutting edge}

Another diamond tool having a round cutting edge was also measured by using the developed on-machine measurement system. Figure 10 shows schematics of two measurement methods with different scanning paths of the optical probe used in this paper.

One method used a raster-like scanning path, in which each scan line was set perpendicular to the tool's edge contour (we call this method "raster-scan method" in this paper) as shown in Fig. 10a. In the method, a priori knowledge on the form of the edge contour would be required to define the scanning path. In this paper, a "pseudo" edge contour was generated by detecting several points on the tool's edge and fitting a circle on them as shown in Fig. 10a.

The other method used a direct scanning path, in which the probe smoothly traced the tool's edge contour (we call this method "direct-scan method" in this paper). Figure 10b shows a schematic of the direct-scan path. The pseudo edge contour calculated in the raster-scan method was also employed in the direct-scan method. The direct-scan method 
Scanning interval: Scanning path (Pseudo tool edge contour) (a)

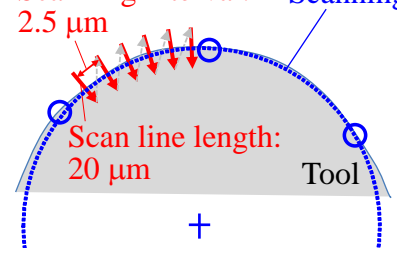

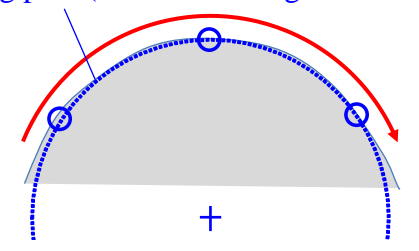

(b)
Figure 10. Schematics of the scanning paths for round cutting edge measurement. (a) Raster-scan method. (b) Direct-scan method.

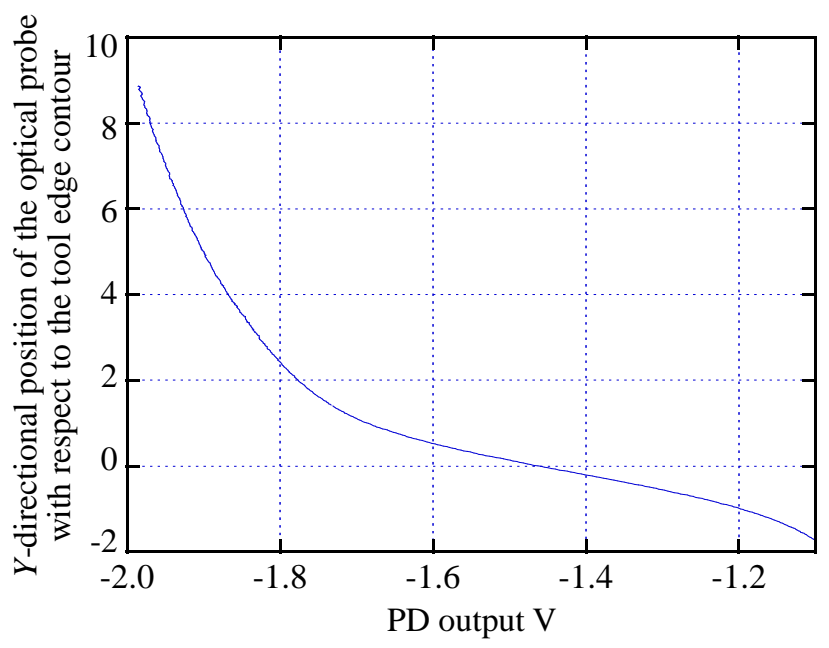

Figure 11. Relationship between the PD output and the $y$ directional relative position of the optical probe with respect to the round cutting edge (nose radius: $0.9 \mathrm{~mm}$ ).

could reduce measurement time. Meanwhile, the optical probe could not measure the tool's form error larger than its diameter when the direct-scan method was applied.

The tool's cutting edge with a nose radius of $0.9 \mathrm{~mm}$ was measured by using the two types of the scanning methods. Measurement range was set to be $0.8 \mathrm{~mm}$. The relationship between the PD output $V$ and the relative position $\delta$ was acquired in advance of the edge contour measurement as shown in Fig. 11. It should be noted that the relation curve in Fig. 11 was different from the one shown in Fig. 7 since the tool's contour of the measurement target is different.

At first, the tool was measured by using the raster-scan method. Figure 12 shows the PD output acquired at each line scan (line $a_{i}-a_{i}^{\prime}$ shown in Fig. 9). The length and number of the sampling points for each scan line were set to be $20 \mu \mathrm{m}$ and 320 points, respectively. The scanning interval along the pseudo edge contour was set to be $2.5 \mu \mathrm{m}$.

Figure 13 shows the measured edge contour, which was calculated from the matrix data of the PD output shown in Fig. 12. When the centre of the micro optical probe is positioned at the edge top of the tool, the intensity $I$ of the optical probe which passes through the tool's edge would be

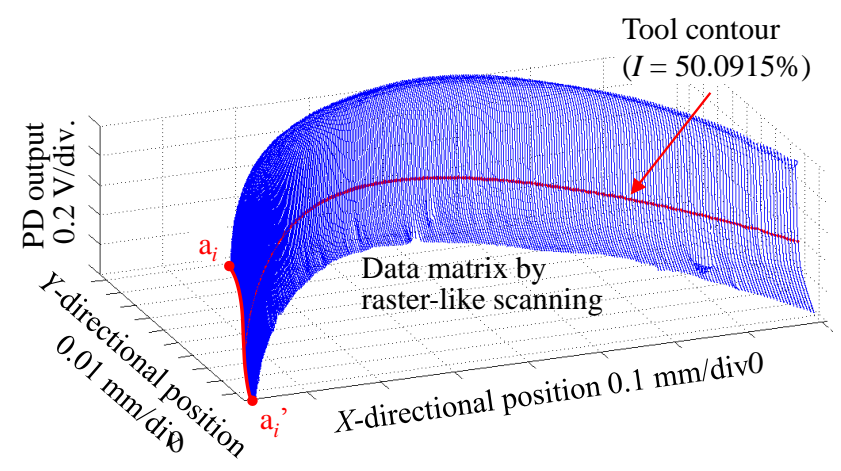

Figure 12. Matrix data of the PD output acquired at each line scan.

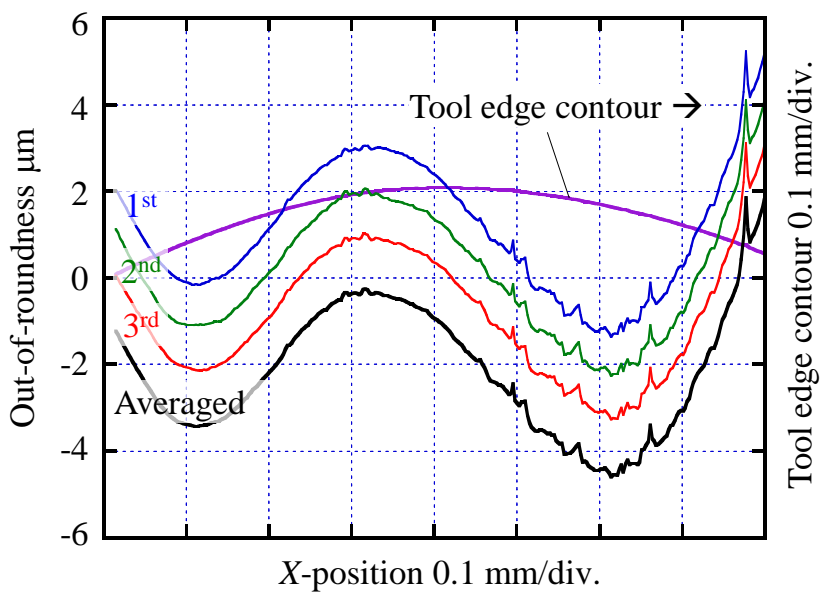

Figure 13. Edge contour and out-of-roundness measured by the raster-scan method.

$50.04 \%$ of that of the whole optical probe, according to the kinematic relationship between the tool's edge (nose radius: $0.9 \mathrm{~mm}$ ) and the micro optical probe (diameter: $2 \mu \mathrm{m}$ ). The tool's edge contour was therefore subtracted from the matrix data in Fig. 12 by using that value. In Fig. 13, out-ofroundness, which is the deviation of the edge contour with respect to a fitted circle, acquired at each measurement is also plotted (each curve was plotted with some offset just for clarity). A good agreement was found among the tool's measured edge contours. Deviations of the out-of-roundness were also shown in Fig. 14. Measurement repeatability was found to be below $0.20 \mu \mathrm{m}$, which was worse than that of the straight cutting edge measurement. One of the main reasons of these results is the long measurement time; it took about $7 \mathrm{~min}$ to carry out measurements with the raster-scan method, and the thermal drift was considered to affect the measurement repeatability.

After that, the edge contour was also measured by using the direct-scan method. Figure 15 shows the edge contour measured by the direct-scan method. In Fig. 15, the scanning path $g(x)$ set for measurement, the deviation of the PD output $\left(V\left(x_{i}\right)\right.$, which will be converted into the relative position 


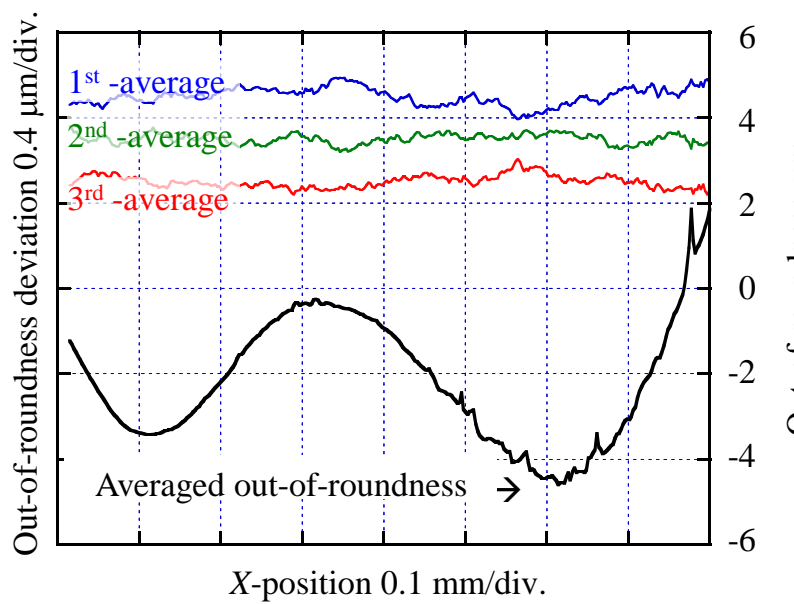

Figure 14. Deviation of the out-of-roundness measured by the raster-scan method.

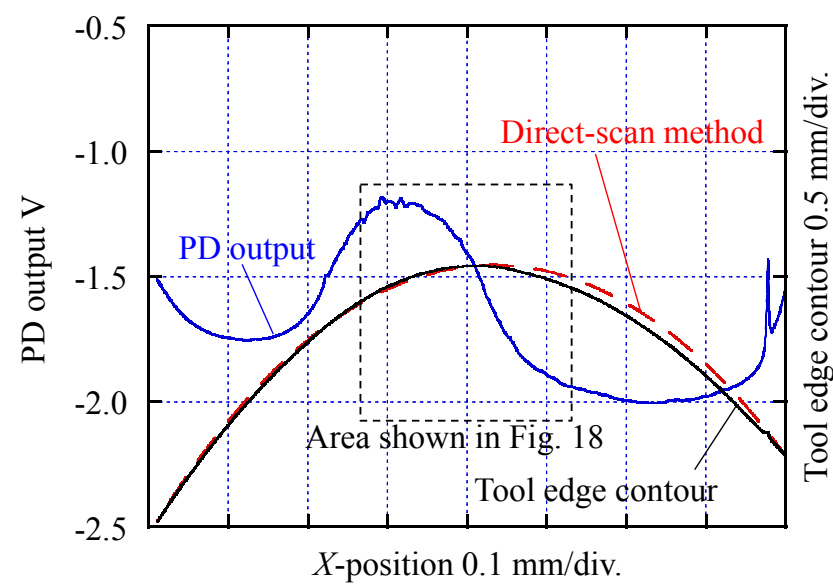

Figure 15. Edge contour of the round cutting edge measured by the direct-scan method.

$\left.\left(\delta x_{i}\right)\right)$ during the scan, and the tool's edge contour $f(x)$ calculated by using Eq. (1) are plotted. Figure 16 shows the out-of-roundness calculated from the measured edge contour shown in Fig. 15. It was also found that the tool's edge contour measured in the two time measurements agreed well with each other. Figure 17 shows the deviation of the outof-roundness. The measurement repeatability in the two time measurements was below $80 \mathrm{~nm}$, which was better than that of the raster-scan method because the required time for the direct-scanning method was relatively shorter $(6 \mathrm{~s})$ than that of the raster-scan method. Figure 18 shows the comparison of the out-of-roundness acquired by the two types of the scanning method. An optical microscopic image of the tool's contour is also shown in Fig. 18. The results by the directscanning method agreed well with the results by the rasterscan method in the region "A" in Fig. 18. Meanwhile, the direct-scanning method could not detect the steeply deviated edge contour in region " $\mathrm{B}$ ", which was well detected by the

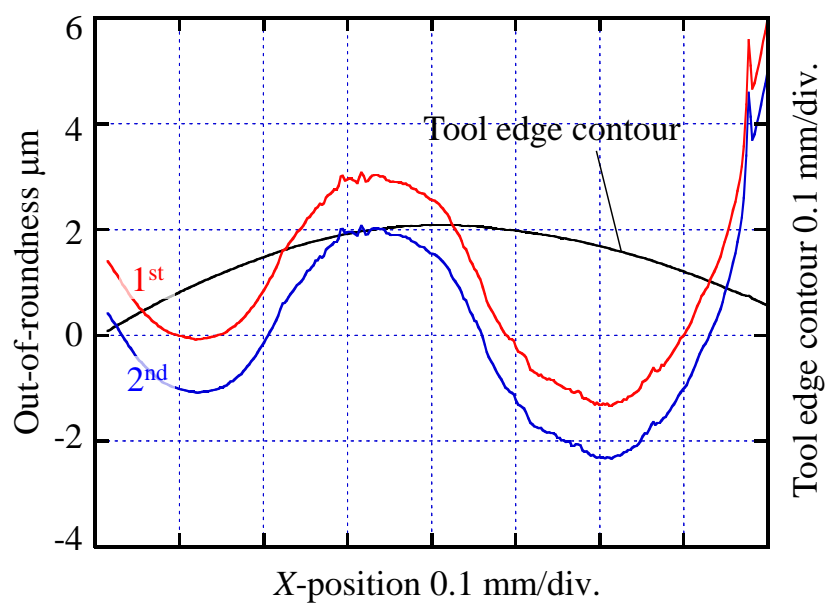

Figure 16. Out-of-roundness measured by the direct-scan method.

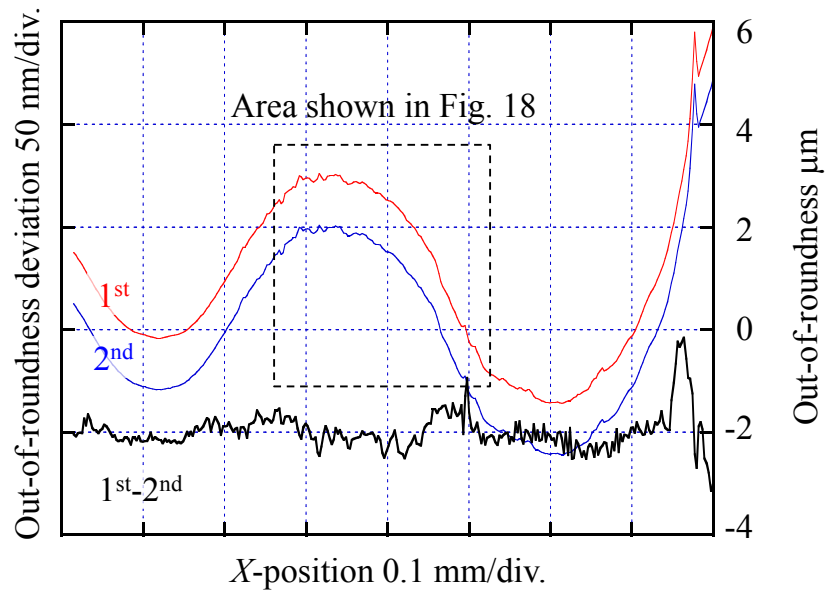

Figure 17. Deviation of the out-of-roundness measured by the direct-scan method.

raster-scan method. The deviation of the tool's edge contour from the scanning path of the optical probe was large and its measurement sensitivity was considered to be degraded, resulting in a spatially averaged form in region " $\mathrm{B}$ " with the direct-scanning method.

\section{Conclusions}

An optical method for edge contour measurement by using a micro optical probe was proposed to evaluate the largescale cutting edge of tools over the measurement range of several millimetres in a short time. An optical setup for the optical micro probe was developed, and its feasibility on the edge contour measurement was investigated in experiments. The stability of the optical micro probe was successfully improved by using the compensation optical system, and the measurement resolution of $10 \mathrm{~nm}$ was achieved. The on-machine measurement system was established by 


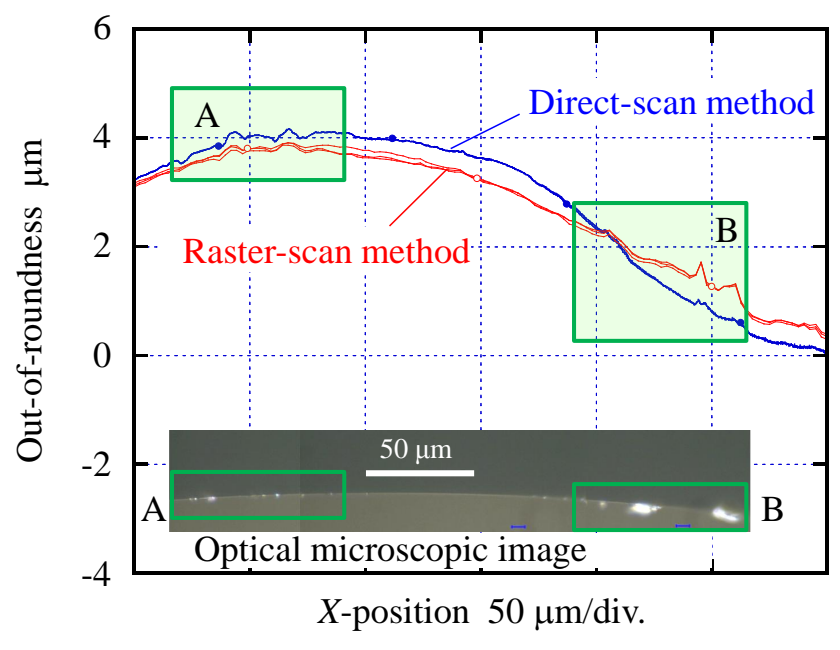

Figure 18. Comparison between the raster-scan method and the direct-scan method.

synchronizing the motion of the $x y z$ slides on the diamond turning machine and the acquisition of PD outputs from the optical probe. Both the straight cutting edge and the round cutting edge of diamond tools were evaluated by the proposed measurement system. For the round cutting edge measurement, both the raster-scan method and direct-scan method were proposed, and the direct-scan method achieved high-speed edge contour measurements in $6 \mathrm{~s}$ for the measurement range of $3.5 \mathrm{~mm}$. Throughout the experiments, the feasibility of the developed measurement system was confirmed. Further detailed analysis on the measurement uncertainty of the developed measurement system should be carried out as a future work.

Acknowledgements. This research was supported by JSPS KAKENHI grant number K2243843 and JST (A-STEP).

Edited by: R. Schmitt

Reviewed by: two anonymous referees

\section{References}

Asai, S., Taguchi, Y., Horio, K., Kasai, T., and Kobayashi, A.: Measuring the very small cutting-edge radius for a diamond tool using a new kind of SEM having two detectors, CIRP Annals, 39, 85-88, 1990.

Brehm, R., van Dun, K., Teunissen, J. C. G., and Haisma, J.: Transparent single-point turning of optical glass, Precision Engineering, 1, 207-213, 1979.

Brinksmeier, E., Riemer, O., Gläbe, R., Lünemann, B., Kopylow, C. V., Dankwart, C., and Meier, A.: Submicron functional surfaces generated by diamond machining, CIRP Annals, 59, 535-538, 2010.

Chen, F. J., Yin, S. H., Huang, H., Ohmori, H., Wang, Y., Fan, Y. F., and Zhu, Y. J.: Profile error compensation in ultra-precision of aspheric surfaces with on-machine measurement, Int. J. Mach. Tool. Manu., 50, 480-486, 2010.

Drescher, J.: Scanning electron microscopic technique for imaging a diamond tool edge, Precision Engineering, 15, 112-114, 1993.

Evansa, C. J. and Bryan, J. B.: "Structured", "Textured" or "Engineered" Surfaces, CIRP Annals, 48, 541-566, 1999.

Fang, F. Z., Zhang, X. D., Weckenmann, A., Zhang, G. X., and Evans, C.: Manufacturing and measurement of freeform optics, CIRP Annals, 62, 823-846, 2013.

Gao, W., Motoki, T., and Kiyono, S.: Nanometer edge profile measurement of diamond cutting tools by atomic force microscope with optical alignment sensor, Precision Engineer, 30, 396-405, 2006.

Gao, W., Asai, T., and Arai, Y.: Precision and fast measurement of $3 \mathrm{D}$ cutting edge profiles of single point diamond micro-tools, CIRP Annals, 58, 451-454, 2009.

Goel, S., Luo, X., Comley, P., Reuben, R. L., and Cox, A.: Brittleductile transition during diamond turning of single crystal silicon carbide, Int. J. Mach. Tool. Manu., 65, 15-21, 2013.

Grosjean, T. and Courjon, D.: Smallest focal spots, Optics Communications, 272, 314-319, 2006.

Jang, S. H., Asai, T., Shimizu, Y., and Gao, W.: Optical analysis of an optical probe for three-dimensional position detection of micro-objects, International Journal of Automation Technology, 5-6, 862-865, 2011.

Jiang, X.: In situ real-time measurement for micro-structured surfaces, CIRP Annals, 60, 536-566, 2011.

Kong, L. B., Cheung, C. F., To, S., Lee, W. B., Du, J. J., and Zhang, Z. J.: A kinematics and experimental analysis of form error compensation in ultra-precision machining, Int. J. Mach. Tool. Manu., 48, 1408-1419, 2008.

Krulewich Born, D. and Goodman, W. A.: An empirical survey on the influence of machining parameters on tool wear in diamond turning of large single-crystal silicon optics, Precision Eng., 25, 24-257, 2001.

Lane, B. M., Shi, M., Dow, T. A., and Scattergood, R.: Diamond tool wear when machining Al6061 and 1215 steel, Wear, 268, 1434-1441, 2010.

Lane, B. M., Dow, T. A., and Scattergood, R.: Thermo-chemical wear model and worn tool shapes for single-crystal diamond tools cutting steel, Wear, 300, 216-224, 2013.

Li, X. P., He, T., and Rahman, M.: Tool wear characteristics and their effects on nanoscale ductile mode cutting of silicon wafer, Wear, 259, 1207-1214, 2005.

Lucca, D. A. and Seo, Y. W.: Effect of tool edge geometry on energy dissipation in ultraprecision machining, CIRP Annals, 42, 83-86, 1993.

Moriwaki, T. and Okuda, K.: Machinability of Copper in UltraPrecision Micro Diamond Cutting, CIRP Annals, 38, 115-118, 1989.

Zong, W. J., Li, D., Sun, T., Cheng, K., and Liang, Y. C.: The ultimate sharpness of single-crystal diamond cutting tools-Part II: A novel efficient lapping process, Int. J. Mach. Tool. Manu., 47, 864-871, 2007.

Zong, W. J., Li, Z. Q., Sun, T., Cheng, K., Li, D., and Dong, S.: The basic issues in design and fabrication of diamond-cutting tools for ultra-precision and nanometric machining, Int. J. Mach. Tool. Manu., 50, 411-419, 2010. 